\title{
Fetal Remains Post-Spontaneous Abortion: Ethical Consideration
}

\author{
Seyed Mohammad Nahidia, d ${ }^{\mathbb{D}}$, Farah Awad ${ }^{\mathrm{b}}$, Drezelle Mills ${ }^{\mathrm{a}}$, \\ Victoria Lewis ${ }^{\mathrm{c}}$, Patrisha Woolard ${ }^{\mathrm{c}}$
}

\begin{abstract}
Disposal of fetal remains as a biohazard material after spontaneous abortion has been the standard practice in many states across the United States of America. In certain states, when a patient requests the fetal remains, it raises a controversy. A proper funeral and burial may be beneficial for patients who suffered from a miscarriage. A patient who is informed of the options available to her after a miscarriage can then decide the most suitable path to deal with such a tragedy. However, the return of the fetal remains to the patient still remains a controversy in certain locations.
\end{abstract}

Keywords: Spontaneous abortion; Miscarriage; Fetal remains; Ethics; Controversy

\section{Introduction}

Spontaneous abortion is defined as the loss of a pregnancy without prior induction before 20 weeks' gestation [1]. Up to $30 \%$ of pregnancies could end in spontaneous abortion, most often due to fetal chromosomal abnormalities and maternal age [1]. Due to the high incidence and sensitive nature of this event, the subject of fetal remains may be a common discussion. Therefore, the ethical issues surrounding such a controversial situation become a point of concern for physicians. Conducting a discussion about such a sensitive matter with patients, who have just recently suffered a miscarriage, may be difficult and must be done so delicately. Patients must be made aware of the options available to them on how to proceed with the fetal remains after a spontaneous abortion. In this case, we highlight the ethical issues that may arise when handling fetal remains after spontaneous abortion.

Manuscript submitted November 4, 2021, accepted November 18, 2021

Published online November 29, 2021

aSt George's University, Grenada, West Indies

bSABA University School of Medicine, The Bottom, Caribbean Netherlands

${ }^{\mathrm{c}}$ Wyckoff Heights Medical Center, Brooklyn, NY 11237, USA

${ }^{\mathrm{d} C}$ Corresponding Author: Seyed Mohammad Nahidi, Wyckoff Heights Medical

Center, 374 Stockholm Street, Brooklyn, NY 11237, USA.

Email: snahidi@sgu.edu

doi: https://doi.org/10.14740/ijcp466

\section{Case Report}

A 14-year-old female, G1P0 15 1/7 weeks pregnant, presented to our institution with complaints of abdominal pain for 3 days. She described the pain as 10/10 constant, stabbing, burning, and non-radiating pain in the right lower abdomen. She reported that the pain worsened with movement, urination, and bowel movement. She also admitted to two episodes of vomiting and decreased appetite. She stated that she has dysuria and constipation. She reported vaginal bleeding that requires one pad an hour to contain. She had recently immigrated to the United States of America without the father of the fetus.

Upon admission, her vitals were only significant of tachypnea. Her hematology laboratory values were significant for white blood cells of $23,000 / \mu \mathrm{L}$, with neutrophils of $91.5 \%$. Her comprehensive metabolic panel (CMP) was significant for albumin of $3.3 \mathrm{~g} / \mathrm{dL}$, sodium of $134 \mathrm{mmol} / \mathrm{L}$, blood urea nitrogen of $3 \mathrm{mg} / \mathrm{dL}$, and creatinine of $0.51 \mathrm{mg} / \mathrm{dL}$. Urine analysis was only remarkable for a large quantity of blood. Appendicitis was suspected and general surgery was consulted.

On hospital day 1 , the patient began to experience intermittent abdominal cramps and a sudden strong urge to urinate, which ended in a bedside spontaneous vaginal delivery. The fetus was not viable. However, the patient requested for the fetus to be brought to her so she can hold it and give it a proper burial.

The patient was scheduled for an appendectomy the next day. The surgery was conducted with no complications and the patient was discharged home on hospital day 4 . The still born fetus was returned to the family and the family initiated plans for a proper funeral and burial.

\section{Discussion}

Spontaneous abortion is defined as the loss of a pregnancy before 20 weeks [1]. During the first trimester, spontaneous abortion of embryonic origin accounts for $80-90 \%$ of miscarriages [1]. Patients with spontaneous complete abortion may present with a history of vaginal bleeding, abdominal pain, and passage of tissue [1].

Labeling embryos and fetal remains as medical waste can be traced from the early 20th century when embryologists and the state established a relationship for scientific studies [2]. 
When it comes to guidelines, second-trimester pregnancy losses are a grey area. Although the fetal body is tangible and more than just tissue, it is not considered the death of a person [3]. A recent study in the UK found various inconsistencies in the legal categorization of a stillborn [3]. It is sometimes acknowledged as a deceased fetus but other times it is merely considered a pregnancy product. They found that legal inconsistencies translated to clinical practice that resulted in patients' beliefs and requests not being prioritized [3]. In the United States of America, the lack of uniformity in the state legislation also impacts how health care institutions recognize miscarriages, informed consent, and fetal remains [4]. For example, in the state of New York, public health law requires a burial permit and disposition of fetal remains after spontaneous abortion only if it occurs after 20 weeks gestation [5]. Before that, there is no legal requirement for the mother to be informed or involved in the disposal process [5]. Recently proposed amendments request that providers are legally required to inform the mother of her right to arrange burial, entombment, or cremation of the remains, regardless of gestational age [5]. Informed consent is the process of disclosing necessary information such as diagnosis, procedural risks, benefits, and alternatives to a competent patient so that they can make an educated and voluntary decision [6]. Informed consent after spontaneous abortion should include a discussion about the available disposal options in detail with the mother [4]. In various studies where parents were interviewed after spontaneous abortion care, most felt they were not educated sufficiently about the alternative options available postmortem. Many reported that they would have preferred tailored discussions with written information to help them make a decision during such an emotional time [7]. Some parents also experienced regret after leaving the remains to health care professionals or not receiving a death certificate [7]. Adequate informed consent may reduce the incidence of these complaints and may provide closure for the grieved family.

In some regions, the legislation mandates that mothers complete documentation and burial/cremation arrangements following spontaneous abortion [8]. This requirement forces active patient involvement and does not take into account that the postpartum period varies for each individual [8]. Formalities that label it as fetal death could add to maternal psychological trauma if it does not align with her views [8]. Researchers recommend that institutions introduce policies that allow collaboration between professionals and patients for respectful disposition [4]. In that case, patients are given the option to let the hospital treat the remains as medical waste, or receive the remains to perform a burial or cremation [4]. If they choose the latter, some extra care such as wrapping or cleaning should be considered [4]. These recommendations allow respectful disposition of fetal remains, which they suggest is an extension of compassionate patient care and allows the healing process to begin [4].

This case sheds light on the lack of guidelines, legislation, and ethical studies available about the matter at hand. The lack of uniformity, as emphasized above, leaves clinicians without guidance. If a patient requests to see the fetus, as the patient did in this case, in a state where a fetus under 20 weeks is labeled as biological waste, there are no guidelines on how to approach this situation and if it should even be allowed. There were no ethical issues in this case as the patient's wishes were honored. However, an ethical consideration arises in the terms of informed consent. Informed consent is a crucial part of medicine. With informed consent, patients are aware of the possible options available to them. Rather than just labeling a fetus under 20 weeks as a biological waste and disposing of it, inform the patient that they have the option to provide the fetus with proper burial. This allows them to have full control and may potentially aid in dealing with the emotions that come after a miscarriage. More studies and ethical research is required to formulate a uniform guideline across all states.

\section{Conclusion}

The fetal remains after a spontaneous abortion are still discarded as biohazard material in most states in the United States of America. However, it may be beneficial to provide the parent, especially the mother, the option of providing a proper funeral and burial to the remains.

\section{Acknowledgments}

None to declare.

\section{Financial Disclosure}

None to declare.

\section{Conflict of Interest}

None to declare.

\section{Informed Consent}

Informed consent has been obtained.

\section{Author Contributions}

Seyed Mohammad Nahidi performed the research including data collection and wrote the paper. Drezelle Mills performed the research including data collection and wrote the paper. Farah Awad performed the research including data collection and wrote the paper. Victoria Lewis reviewed the case report and provided expert clinical knowledge to revise critically. Patrisha Woolard was the pediatrics attending, reviewed the case report, and provided expert clinical knowledge to revise critically.

\section{Data Availability}

The authors declare that data supporting the findings of this study are available within the article. 


\section{References}

1. Alves C, Rapp A. Spontaneous Abortion. In: StatPearls. Treasure Island (FL), 2021.

2. Morgan LM. "Properly disposed of": a history of embryo disposal and the changing claims on fetal remains. Med Anthropol. 2002;21(3-4):247-274.

3. Middlemiss A. Pregnancy remains, infant remains, or the corpse of a child? The incoherent governance of the dead foetal body in England. Mortality. 2021;26(3):299-315.

4. Levang E, Limbo R, Ziegler TR. Respectful Disposition After Miscarriage: Clinical Practice Recommendations. MCN Am J Matern Child Nurs. 2018;43(1):19-25.

5. A.B. 5826, 2021 Biennium, 2021 Reg. Sess. (NY. 2021). https://www.nysenate.gov/legislation/bills/2021/a5826.

6. Cocanour CS. Informed consent-It's more than a signature on a piece of paper. Am J Surg. 2017;214(6):993997.

7. Ellis A, Chebsey C, Storey C, Bradley S, Jackson S, Flenady V, Heazell A, et al. Systematic review to understand and improve care after stillbirth: a review of parents' and healthcare professionals' experiences. BMC Pregnancy Childbirth. 2016;16:16.

8. Joseph KS, Basso M, Davies C, Lee L, Ellwood D, Fell $\mathrm{DB}$, Fowler D, et al. Rationale and recommendations for improving definitions, registration requirements and procedures related to fetal death and stillbirth. BJOG. 2017;124(8):1153-1157. 